\title{
Effective Corner Matching for Transformed Image Identification
}

\author{
Mohammad Awrangjeb and Guojun Lu \\ Gippsland School of Information Technology, Monash University, \\ Churchill Vic 3842, Australia \\ \{Mohammad.Awrangjeb, Guojun.Lu\}@infotech.monash.edu.au
}

\begin{abstract}
There are many applications, for example image copyright protection, where transformed images of a given test image need to be identified. The solution to the identification problem consists of two main stages. In stage one, certain representative features are detected for all images. In stage two, the representative features of the test image and the stored images are compared to identify the transformed images for the test image. We have reported the technique to extract robust representative features - corners - in our previous work [1]. This paper will focus on our stage-two work on effective corner matching technique for transformed image identification. Experimental results show that the proposed corner matching technique is very much effective in identifying the transformed images for a given test image.
\end{abstract}

Key words: corner detection, corner matching, image matching, transformed image identification

\section{Introduction}

In many applications, such as image copyright protection [2], one common problem is to identify images which may undergo unknown transformations. We can define this common problem as the transformed image identification (TII) where the goal is to identify both the geometric transformed and the signal processed images for a given test image. So the TII is different from the conventional content-based image retrieval (CBIR) [3], where all images having the same or similar semantic features, e.g., 'red car', 'rose', are considered relevant to each other.

The TII consists of two main stages. The first stage is feature detection and representation where a set of representative features are detected and represented for all images. We have reported the technique to detect robust representative features - corners - in our previous work [1]: the affine-resilient curvature scalespace (ARCSS) corner detector. It detects corners on planar curves and offers better performance in terms of both average repeatability and localization error than the existing CSS detectors $[4,5]$ under geometric transformations. Each corner is represented with information such as its position, absolute curvature 
value, corresponding curve number, and affine-lengths between this corner and other corners on the same curve.

The second stage, which is feature matching, is the focus of this paper. In this stage, the representative features of the test image and the stored images are compared to identify those database images which are geometric transformed and signal processed images of the same original image for a given test image. Feature matching solutions in the literature can be broadly categorized into two: those which use the local neighborhood of each feature for matching [7] and those which use feature information like its curvature but do not use neighbor intensity values for matching $[6,8-13,16]$. The second category solutions can also be divided into two groups: model-based [10-13] and model-free $[6,8,9,16]$ techniques. In model-based feature matching techniques, the correspondence between two point-sets is established with the help of some predefined (given) object models. In contrast, in model-free feature matching techniques, such predefined object models are absent and, therefore, the correspondence between the sets should be made directly without the help of the models. As a result, the model-free solutions are harder to design than their model-based counterparts. Moreover, the model-based techniques are application specific e.g., object recognition, while the model-free techniques are more general and can be used for copyright protection [2].

The proposed corner matching solution resides in the model-free group as it does not use any predefined object model to establish corner correspondences between two corner sets. The proposed matching technique is robust to both geometric transformations like rotation and scaling and signal processing like compression and noising. It can be used with any contour-based corner detector. Particularly, here we will use it with our previously proposed ARCSS corner detector [1], which makes necessary information, such as curvatures at the corner points and affine-lengths between corners, available to the matching algorithm.

We obtain the TII performance using the precision-recall graph and compare with the existing gray-scale histogram (GSH) matching technique [3]. In TII the database images which are originated (either geometric transformed or signal processed or both) from the same original image as the test image reside in the same group and are considered relevant to each other. Consequently, many existing CBIR techniques [3] may not be applicable to TII, since the goal of CBIR is different from that of TII. The GSH matching, which is a global feature (intensity frequency) based CBIR technique, is very much robust to geometric transformations [3] and, therefore, is chosen to compare with the proposed corner matching technique while both of them are applied to TII.

Note that the corner matching performance (how many repeated corners the matching technique can find) of the proposed corner matching technique was published in [17]. Experimental results showed that the proposed matching technique obtained maximum number of true corner correspondences. In this paper we have investigated its performance to identify the transformed images for a given test image. We have also proposed different strategies to reduce the search-space. 


\section{Proposed matching technique}

Absolute curvatures of corners may change due to affine transformations; however, for many corners they either remain unaffected or change slightly. Moreover, the affine-length of a planar curve and the area of the triangle, consisting of three corners as vertices, are relatively invariant [15] to geometric transformations. In the proposed corner matching technique, for each corner we use its position, absolute curvature value, corresponding curve number, and affine-lengths between this corner and other corners on the same curve. The iterative matching procedure first finds three corner matches between a test image and a database image within a specific absolute curvature difference. If the three matching corners are non-collinear on each image and the ratio of areas of corresponding triangles in both the images is within a specific range, it obtains the geometric transformation parameters between these triangles. Then it transforms all corners in the database image using the estimated transformation matrix and matches with the test corner set. In rest of the paper, we will call the proposed matching technique as affine-length and triangular area (ALTA) matching technique.

\subsection{Relative Invariants}

We define the term relative invariance with respect to the two-dimensional (2D) space according to the classical geometric invariant theory from [15] as

Definition 1. For a given $2 D$ space $\Omega$ and a given transformation group $\chi$ active on $\Omega$, the function $f(x, y)$ is relatively invariant to $\chi$ if and only if we have the transformed function $f_{a}(x, y)=g(f(x, y))=h(\operatorname{det}(g)) . f(x, y), \forall(x, y) \in \Omega$ and $\forall g \in \chi$, where $\operatorname{det}(g)$ denotes the determinant of the transformation matrix for $g$ and $h(\operatorname{det}(g))$ is some function of $\operatorname{det}(g)$.

When $h(\operatorname{det}(g))=1$, the above relation becomes absolutely invariant. The affine-length between two points $P_{1}$ and $P_{2}$ of a planar curve $(x(t), y(t))$ is [1]

$$
\tau=\int_{P_{1}}^{P_{2}} \sqrt[3]{\dot{x}(t) \ddot{y}(t)-\ddot{x}(t) \dot{y}(t)} d t
$$

where $\dot{x}(t)$ and $\dot{y}(t)$ are first and $\ddot{x}(t)$ and $\ddot{y}(t)$ are second order derivatives with respect to the arbitrary parameter $t$. We can show that the affine-length of a transformed curve is

$$
\tau_{a}=\left(s_{x} s_{y}\right)^{1 / 3} \cdot \tau .
$$

where $s_{x}$ and $s_{y}$ are scaling factors along $x$ and $y$ axis. The relation in (2) shows that the affine-length is absolutely invariant to rotation and translation, but relatively invariant to scaling.

The area $\Delta$ of a triangle with vertices $v_{1}=\left(x_{1}, y_{1}\right), v_{2}=\left(x_{2}, y_{2}\right)$, and $v_{3}=\left(x_{3}, y_{3}\right)$, when $v_{1}$ is shifted to the origin, is

$$
\Delta\left(v_{1}, v_{2}, v_{3}\right)=\frac{1}{2}\left|\left(x_{2}-x_{1}\right)\left(y_{3}-y_{1}\right)-\left(x_{3}-x_{1}\right)\left(y_{2}-y_{1}\right)\right| .
$$




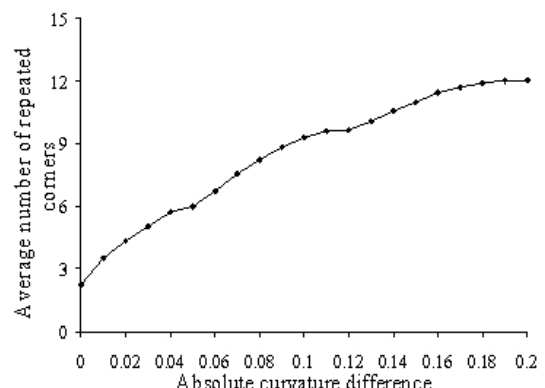

(a)

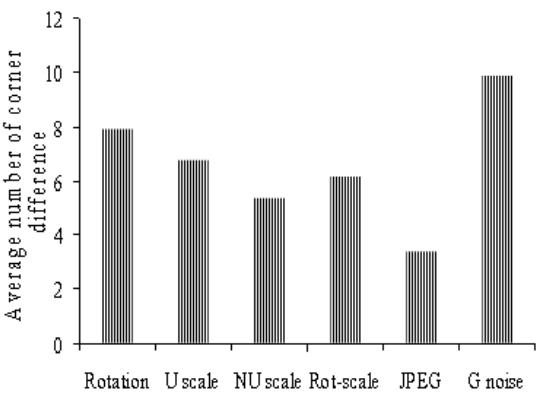

(b)

Fig. 1. Performance analysis of the ARCSS detector [1]: (a) average number of repeated corners and (b) average number of corner difference between original and test images.

Similar to (2), we can derive the area of a transformed triangle as

$$
\Delta_{a}\left(v_{1 a}, v_{2 a}, v_{3 a}\right)=s_{x} s_{y} \cdot \Delta\left(v_{1}, v_{2}, v_{3}\right),
$$

which implies that the triangular area is also relatively invariant to scaling.

In the proposed matching technique, we use the affine-length between corners on the same curve to find corner matches and the triangular area to reduce the search-space. However, comparing (2) and (4), it is evident that the affine-length is less sensitive to geometric transformations than the triangular area, because in the former the power of $s_{x} s_{y}$ is $\frac{1}{3}$ but in the later it is 1 . This means that in large scaling factors the search-space will not be reduced much. Therefore, we introduce other strategies to reduce the search-space (see Section 2.3).

\subsection{Iterative Matching Procedure}

A subset $\left\{I_{p}\right\}$ of the database images is selected (see Section 2.3a) as the set of possible transformed images for a given test image $I_{q}$. For each pair of images $\left(I_{p}, I_{q}\right)$, the ALTA iterative corner matching procedure is as follows.

Algorithm 1 ALTA corner matching algorithm.

Input: Two corner sets $C_{p}$ and $C_{q}$ of $I_{p}$ and $I_{q}$ respectively. Each corner is associated with its position, absolute curvature value, corresponding curve number, and the affine-length of its predecessor on the same curve.

Output: Information about image matches.

1. Set curvature difference threshold $T_{C D}=0.2$, minimum corner matches $m=3$, and output $O_{p q}=\varnothing$ where $\varnothing$ denotes the empty set.

2. Find the set of candidate corner matches $C_{c}$ between $C_{p}$ and $C_{q}$ with curvature difference less than or equal to $T_{C D}$.

3. For each candidate match $\left(c_{p}, c_{q}\right)$ in $C_{c}$, add new candidate match $\left(c_{p n}, c_{q n}\right)$ to $C_{c}$ if the affine-length ratio of corresponding curve segments $c_{p} \rightsquigarrow c_{p n}$ and $c_{q} \rightsquigarrow c_{q n}$ is within the range $[l, h]$. 
4. For (next) three non-collinear candidate matches $\left(c_{p 1}, c_{q 1}\right),\left(c_{p 2}, c_{q 2}\right)$, and

$\left(c_{p 3}, c_{q 3}\right)$ in $C_{c}$, if the ratio of areas of triangles $\Delta_{p}\left(c_{p 1}, c_{p 2}, c_{p 3}\right)$ and $\Delta_{q}\left(c_{q 1}, c_{q 2}, c_{q 3}\right)$ is within the range $\left[l_{a}, h_{a}\right]$, find $A_{R S}$ and $T$.

5. Transform all corners in $C_{p}$ using $A_{R S}$ and $T$; i.e., $C_{p t}=A_{R S} . C_{p}+T$.

6. Find the set of corner matches $C_{m}$ between $C_{p t}$ and $C_{q}$.

7. If $\left(\left|C_{m}\right|>m\right), O_{p q}=O_{p q} \cup\left[p, q, C_{m}, A_{R S}, T\right]$. Go to step 4.

Fig. 1(a) shows that the ARCSS detector [1] has on the average more than 12 repeated corners within curvature difference 0.2 ; however, we need only 3 of them to estimate the geometric transformation parameters. Therefore, in step 1 of Algorithm $1, T_{C D}$ is set to 0.2 experimentally, because for larger value the procedure becomes more expensive and for smaller value we may miss some relevant images. We also set $m$ to 3 in step 1, because for any three non-collinear candidate matches there are already 3 matches. In step 2, we find the candidate corner matches within the curvature difference $T_{C D}$. In step 3 , corners $c_{p}$ and $c_{p n}$ should have the same curve number in $C_{p}$ and corners $c_{q}$ and $c_{q n}$ should have the same curve number in $C_{q}$. In this step, we add other possible candidate matches for each candidate match found in step 2 . We only consider those curves which have more than 1 corners and at least 1 of them have already been selected as candidate matches in the previous step. For adding a new candidate match, we only consider affine-length ratio between this corner and an already added candidate corner on the same curve regardless of their curvature values. The ranges $[l, h]$ of the affine-length ratio in step 3 and $\left[l_{a}, h_{a}\right]$ of triangular area ratio in step 4 are set according to (2) and (4) respectively. While finding corner matches in step 6 , we allow a maximum mean-square-error of 9, i.e., $R M S E \leq 3$ pixels.

\subsection{Speeding up the Identification}

The loop of steps 4-7 may make the procedure computationally expensive. We apply the following measures to speed up.

a. Selecting the possible transformed images: Fig. 1(b) shows that the average difference in corner numbers between original and test images is maximum 11 (for Gaussian noise) by the ARCSS corner detector [1]. Therefore, a database image with 20 corners may not be a possible transformed image for a query image $I_{q}$ with 80 corners, and vice versa. The subset $\left\{I_{p}\right\}$ of the database images are selected as possible transformed images of $I_{q}$ such that $a b s\left(\left|C_{p}\right|-\left|C_{q}\right|\right) \leq 25$. Consequently, for a given test image, we do not execute the corner matching procedure for all database images.

b. Pre-processing: Although more than 3 corners can be detected on each curve by the ARCSS detector, we need only 3 true corner matches to obtain the geometric transformation. Therefore, a pre-processing is carried out on the input corner sets $C_{p}$ and $C_{q}$ separately so that maximum 3 corners will have the same curve number in each input corner set, since we need only 2 more true matches along with a possible true match found in step 2. This pre-processing significantly reduces the number of false new candidate match additions in step 3 
for each false candidate match found in step 2 and, hence, speeds up the iterative procedure by reducing the search-space.

c. Matching the triangular area: In step 4, the condition of the ratio of areas of two triangles to be in a specific range also reduces the number of tests in the loop with the false candidate corner-matches. However, (4) shows that the area of a triangle increases with the increase of scaling factors, i.e., when $s_{x} s_{y}>1$. Because false triangular area matches will also increase when the upper-limit of the range $\left[l_{a}, h_{a}\right]$ increases with large scaling factors. Consequently, the search-space may not be reduced much in this situation using the triangular area matching.

d. Using the hash table: The procedure is further speeded up by using a simple hash table for storing the combinations of candidate corner matches already visited in step 4. Assuming that there will be maximum 99 (two digits) strong corners detected in each image, each key $k$ stored in the hash table is 12 digits long (6 vertices of two triangles) where consecutive two digits denote a corner number (vertex). We use the division method [14] for mapping the key $k$ into one of $i$ slots in the hash table by taking the remainder of $k$ divided by $i$. That means, the hash function is $h(k)=k \bmod i$. The hash table can be effectively and efficiently implemented using the MATLAB cell data structure where each slot in the hash table is a variable-length one-dimensional array, initially set to zero size. We store all keys mapped to a particular slot in order of their arrival. In order to reduce the collisions, i.e., number of keys mapped to the same slot, the value of $i$ is chosen to be a prime that is not too close to the exact power of 2. Again assuming maximum total 10,000 keys to store, the total number of slots $i$ is chosen to be a prime number 3067, so that on average 3 keys are mapped in each slot, which makes the search very efficient. As an example of calculating the hash function, suppose corner numbers 12,25 , and 50 in the database image and 34,65, and 84 in the query image are visited during an iteration of loop 4-7. Hence, the key $k=122550346584$ is stored in the slot number $h(k)=k \bmod 3067=942$ of the hash table.

e. Selecting the top corners: If all corners are considered, it may make the procedure still expensive depending on the number of corners in $I_{p}$ and $I_{q}$. Fortunately, by selecting only top (say, 15) corners with higher curvature values, it not only speeds up the iteration but also offers better performance (see detail in Section 3).

\section{Performance Study}

For evaluating the identification performance, we applied ALTA and GSH matching [3] techniques for TII and compare them in the precision-recall graph. We used a large database of total 1700 images (including original and transformed).

Database In the identification database, we had 100 different original $512 \times 512$ gray-scale images including 'Lena', 'Elaine', and 'Boat' [18] and their 1600 transformed images of five categories: 
i) rotation at 4 different angles $\theta$ in $\left[5^{\circ} 20^{\circ}\right]$ at $5^{\circ}$ apart;

ii) uniform (U) scale factors $s_{x}=s_{y}$ in $[0.851 .15]$ at 0.05 apart, excluding 1.0;

iii) combined transformations (rot.-scale): $\left[\theta, s_{x}, s_{y}\right]=\left[5^{\circ}, 0.8,1.2\right]$ and $\left[10^{\circ}, 0.7,1.1\right]$;

iv) JPEG lossy compression at quality factors 20 and 25 ; and

v) zero mean white Gaussian $(\mathrm{G})$ noise with noise-variances 0.005 and 0.01 .

We had 5 queries for each original image: rotation $10^{\circ}$, uniform scale factor 1.05 , combined transformation $\left[5^{\circ}, 0.8,1.2\right]$, lossy JPEG compression with quality factor 20, and Gaussian noise with variance 0.005 . Therefore, we had total 100 groups of images in this database, each group consists of 17 relevant images (16 transformed and 1 original) for a corresponding query, and in total 1700 images in the database. All images within each group are considered relevant to each other. We also cropped all rotated images so that the outer black parts were disappeared. This must diminish the bias of large black regions in rotated images for the GSH matching technique [3].

Evaluation Metrics The database images which were originated from the same original image as the query image using some geometric transformations or signal processing are considered relevant to each other. We used precision and recall [3] collectively to measure the identification performance. Recall measures the system capacity to retrieve the relevant images from the database. It is defined as the ratio between number of retrieved relevant images $r$ and total number of relevant images $T$ (group size) in the database:

$$
\text { Recall }=\frac{r}{T} .
$$

Precision measures the retrieval accuracy. It is defined as the ratio between $r$ and number of retrieved images $R$ :

$$
\text { Precision }=\frac{r}{R} \text {. }
$$

In practice, the performance of an information retrieval system is presented using the precision-recall graph, where the higher the precision at a given recall value the better the performance of the retrieval system [3].

Results and Discussions We have evaluated and compared the identification performance for both GSH matching and ALTA corner matching techniques. While the ALTA matching used the number of corner matches between the query and database images to rank the retrieved images, the histogram matching used the normalized $L-1$ distance [3]. In case of ALTA matching, we considered two cases - when all corners were considered and when only top 15 corners with the highest curvature values were considered. We selected top 15 corners based on two observations. First, if more than 15, say 20 or more, were selected, the procedure became more expensive. Second, if less than 15, say 10 or less, were selected, almost the same number of corner matches were found for both relevant 
and irrelevant images in the database. Choosing top corners not only reduced the computational complexity but also improved the performance. Because if all the corners are used, there might be high number of corner correspondences between irrelevant images. By selecting top 15 corners based on the highest curvature values before establishing the corner correspondence decreased the probability of establishing such false corner correspondences.

Fig. 2(a)-(e) present the identification performance by the proposed ALTA matching and the existing GSH matching [3] techniques under five different queries on the identification database. The ALTA matching technique offered better performance than the GSH matching technique in most of the cases. Nonetheless, for lower recall values the GSH matching outperformed the ALTA matching, specially in queries comprising scaling (see Fig. 2(b)-(c)). However, for higher recall values while the precision of the ALTA matching decreased slightly, that of GSH matching dropped significantly in all queries. This might be due to two reasons. First, scaling may preserve the ratio of gray-scale intensities but in higher recall values different images may have the same histogram. Second, since ALTA matching considered all the detected corners, some irrelevant images may be retrieved.

Nevertheless, instead of considering all the detected corners, when we considered only top few corners from each image based on the highest curvature values, the TII performance in the precision-recall graph increased significantly. Moreover, the computational cost fell significantly as the number of corners decreased. Fig. 2(a)-(e) show that the ALTA matching with top 15 corners outperformed the GSH matching in most of the cases. The average performance in five queries is shown in Fig. 2((f). The precision of the ALTA matching procedure is above $90 \%$ which is almost the same in all recall values, but that of GSH matching dropped to $40 \%$ at $100 \%$ recall.

\section{Conclusions}

The proposed ALTA corner matching method can be used with any contourbased corner detector depending on the availability of the required information for establishing the corner correspondence. It takes the advantage of the affinelength invariance between corners on the same curve. It also uses the absolute curvature values which either remain unaltered or change slightly under geometric transformations. It follows different strategies to reduce the search-space.

While applying for TII, the ALTA matching technique offered much better retrieval performance in the precision-recall graph than the GSH matching technique [3]. The average precision (see Fig. 2(f)) by the ALTA matching is always above $90 \%$ under all recall values and, therefore, it is evident that the proposed matching technique could be successfully exploited in many computer vision applications including image copyright protection [2].

However, we observed that in spite of taking measures discussed in Section 2.3 , the proposed ALTA matching technique required more time than the existing GSH technique when both were applied to TII. Future works include investigat- 
ing the more time-efficient corner matching technique while maintaining at least the same performance.

\section{References}

1. Awrangjeb, M., Lu, G.: An Affine Resilient Curvature Scale-space Corner Detector. In Proc. Int. Conf. on Acoustics, Speech, and Signal Proces.. Vol. 1. Hawaii, USA (2007) 1233-1236.

2. Awrangjeb, M., Murshed, M.: Robust Signature-based Geometric Invariant Copyright Protection. In Proc. Int. Conf. on Image Proces. Atlanta, USA (2006) 19611964.

3. Lu, G.: Multimedia Database Management Systems. Artech House Inc., Norwood (1999).

4. Mokhtarian, F., Suomela, R.: Robust Image Corner Detection Through Curvature Scale Space. IEEE Trans. on Pat. Anal. and Mach. Intel., Vol. 20(12). (1998) 13761381.

5. Mokhtarian., F., Mohanna, F.: Enhancing the Curvature Scale Space Corner Detector. In Proc. Scandinavian Conf. on Image Analysis. (2001) 145-152.

6. Zhou, D., Li, G., Liu, Y.: Effective Corner Matching Based on Delaunay Triangulation. In Proc. Int. Conf. on Robotics and Automation. Vol. 3, (2004) 2730-2733.

7. Matas, J., Chum, O., Urban, M., Pajdla, T.: Robust Wide Baseline Stereo From Maximally Stable External Regions. In Proc. British Machine Vision Conference. (2002) 384-393.

8. Jung, I., Lacroix, S.: A Robust Interest Points Matching Algorithm. In Proc. Int. Conf. on Computer Vision. Vol. 2, (2001) 538-543.

9. You, J., Pissaloux, E., Cohen, H.: A Hierarchical Image Matching Scheme Based on the Dynamic Detection of Interesting Points. In Proc. Int. Conf. on Acoustics, Speech, and Signal Processing. Vol. 4, (1995) 2467-2470.

10. Lee, K., Kim, Y., Myung, H., Kim, J., Bien, Z.: A Corner Matching Algorithm with Uncertainty Handling Capability. In Proc. Int. Conf. on Fuzzy Systems. Vol. 3, (1997) 1469-1474.

11. Rutkowski, W.: Recognition of Occluded Shapes Using Relaxation. Comp. Graph. and Img. Process. Vol. 19(2), (1982) 111-128.

12. Horaud, R., Skordas, T.: Stereo Correspondence Through Feature Grouping and Maximal Cliques. IEEE Trans. on Pat. Anal. and Mach. Intel. Vol. 11(11), (1989) 1168-1180.

13. Nasrabadi, N., Li, W.: Object Recognition by a Hopfield Neural Network. IEEE Trans. on Sys., Man and Cyb. Vol. 21(6), (1991) 1523-1535.

14. Cormen, T., Leiserson, C., Rivest, R.: Introduction to Algorithms. The MIT Press, London (1999).

15. Klein, F.: Elementary Mathematics From an Advanced Standpoint: Geometry. The Macmillan Company, New York (1939).

16. Hopcroft, J., Huttenlocher, D.: On Matching Planar Points Sets Under Affine Transformations. Technical Report TR. 89-986, Department of Computer Sceince, Cornell University, New York (1989).

17. Awrangjeb, M., Lu, G.: A Robust Corner Matching Technique. In Proc. Int. Conf. on Multimedia and Expo. Beijing, China (2007) 1483-1486.

18. http://personal.gscit.monash.edu.au/ awran/pcm.html (2007). 


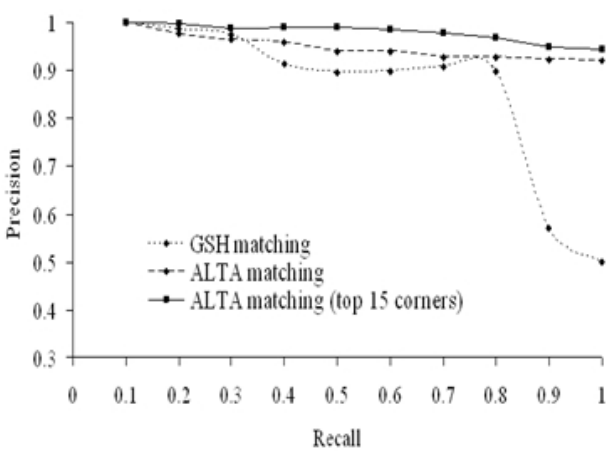

(a)

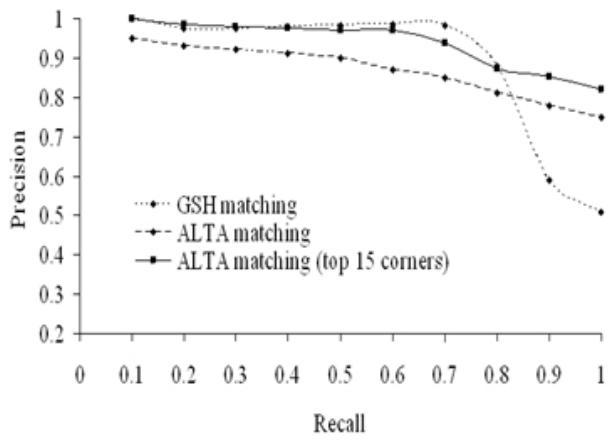

(c)

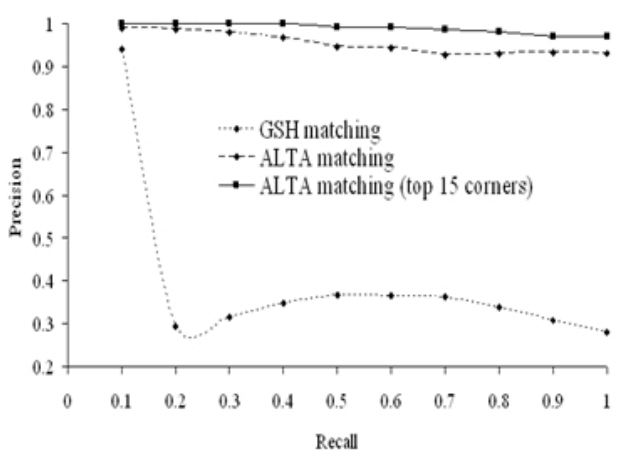

(e)

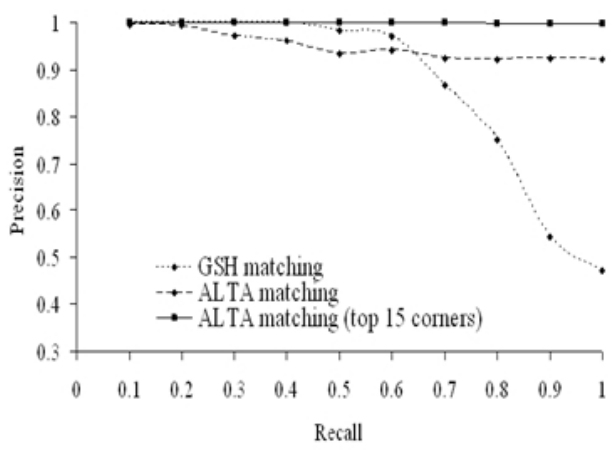

(b)



(d)

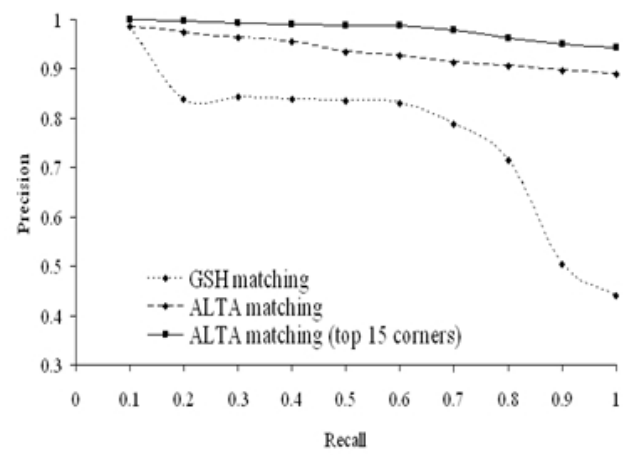

(f)

Fig. 2. The TII performance in (a) query 1: rotation $\left(\theta=10^{\circ}\right)$, (b) query 2: uniform scale $\left(s_{x}=s_{y}=1.05\right)$, (c) query 3: rotation-scale $\left(\theta=10^{\circ}, s_{x}=0.8, s_{y}=1.2\right)$, (d) query 4: JPEG (quality factor 20), (e) query 5: zero mean Gaussian noise (noisevariance $=0.005)$, and (f) average in all five queries (a)-(e). 